\title{
Significados de convivencia: una comprensión desde la dinámica cotidiana que los promueve
}

Ana Lucía Sánchez-Villafañe*, Jhon Jairo Quiceno-Pérez ${ }^{\star *}$

Especialista en Desarrollo Comunitario. Profesora, Facultad de Psicología, Universidad Cooperativa de Colombia, Cali, Colombia.

Correo electrónico:

ana.sanchezv@campusucc.edu.co

** Psicólogo. Profesor, Facultad de Psicología, Universidad Cooperativa de Colombia, Cali, Colombia. Correo electrónico:

jhon.quiceno@campusucc.edu.co

Recibido: 20 de enero del 2016

Aprobado: 8 de marzo del 2016

Cómo citar este artículo: SánchezVillafañe, A. L. y Quiceno-Pérez, J. J. (2016). Significados de convivencia: una comprensión desde la dinámica cotidiana que los promueve. Pensando Psicología, 12(19), 55-67. doi: http://dx.doi.org/10.16925/ pe.v12i19.1328

\section{Resumen}

Introducción: la convivencia se constituye en un factor primordial en el establecimiento de relaciones sociales. Infortunadamente, en Colombia se ha visto fracturada por altos índices de violencia que han exigido el desarrollo de políticas públicas, muchas de ellas impuestas y alejadas de las necesidades de las comunidades. Objetivo: por tal motivo, se hace indispensable que sean las comunidades quienes definan y signifiquen la convivencia, a fin de generar propuestas más cercanas a la realidad. En este sentido, se pretende identificar los significados que en relación con las prácticas de convivencia comunitaria construye un grupo de líderes. Metodología: se realizó un estudio cualitativo-descriptivo con diseño etnográfico, en un grupo de cinco (5) líderes. Resultados: la convivencia fue definida como aquellos procesos que hacen posible la construcción y el establecimiento de acuerdos que garantizan la consolidación de buenas relaciones en la comunidad y evitar los conflictos. Esta concepción solo puede operar en la medida en que la solidaridad sea el valor que guíe y oriente las relaciones, y que la participación comunitaria sea efectiva. Sin embargo, la conducta de solidaridad termina en el acto mismo de ayuda, y no se extiende a otras experiencias comunitarias que promuevan la cohesión social. Conclusiones: se evidenció una visión idealizada de convivencia en torno a una anhelada solidaridad. En la cotidianidad priman dinámicas focalizadas en el individualismo y la apatía, debido a la desconfianza mutua, a la politización de las relaciones y a una visión asistencialista que impide la transformación desde un proyecto colectivo.

Palabras clave: convivencia, comunidad, liderazgo y solidaridad, significados. 


\title{
Meanings of Coexistence: An Understanding From the Daily Dynamics Promoting Them
}

\begin{abstract}
Introduction: Coexistence constitutes a major factor in establishing social relations. In Colombia, unfortunately, it has been fractured by high rates of violence that have required the implementation of public policies, many of them imposed and away from the needs of communities. Objective: For this reason, it is essential that communities define and give meaning to coexistence in order to make proposals closer to reality. In this regard, the article seeks to identify the meanings that, in relation to the practices of community coexistence, are built by a group of leaders. Methodology: A qualitative descriptive study with an ethnographic design was conducted in a group of five (5) leaders. Results: Coexistence was defined as those processes that make it possible to construct and establish agreements that guarantee the consolidation of good relations in the community and avoid conflict. This concept can only operate to the extent that solidarity becomes the value guiding relationships and that community participation is effective. However, this solidarity behavior finishes in the act of helping itself and does not extend to other community experiences that promote social cohesion. Conclusions: An idealized vision of coexistence around a desired solidarity was evident. In everyday life, dynamics focused on individualism and apathy take precedence, because of mutual distrust, politicization of relations and a bandaid vision that prevents transformation from a collective project.
\end{abstract}

Keywords: coexistence, community, leadership and solidarity, meanings.

\section{Significados de convivência: uma compreensão a partir da dinâmica cotidiana que os promove}

\section{Resumo}

Introdução: a convivência se constitui num fator primordial no estabelecimento de relações sociais. Infelizmente, na Colômbia, tem sido vista prejudicada por altos índices de violência que vêm exigindo o desenvolvimento de políticas públicas, muitas delas impostas e afastadas das necessidades das comunidades. Objetivo: por esse motivo, faz-se indispensável que sejam as comunidades as que definam e signifiquem a convivência a fim de gerar propostas mais próximas à realidade. Nesse sentido, pretende-se identificar os significados que, com relação às práticas de convivência comunitária, um grupo de líderes constrói. Metodologia: realizou-se um estudo qualitativo-descritivo com desenho etnográfico, num grupo de cinco líderes. Resultados: a convivência foi definida como aqueles processos que fazem possível a construção e o estabelecimento de acordos que garantem a consolidação de boas relações na comunidade e evitam os conflitos. Essa concepção só pode operar na medida em que a solidariedade seja o valor que guie e oriente as relações e que a participação comunitária seja efetiva. Contudo, o comportamento solidário termina no ato em si de ajuda e não se estende a outras experiências comunitárias que promovam a coesão social. Conclusões: evidenciou-se uma visão idealizada de convivência em torno de uma anelada solidariedade. Na cotidianidade, priorizam-se dinâmicas focalizadas no individualismo e na apatia, devido à desconfiança mútua, à politização das relações e a uma visão assistencialista que impede a transformação a partir de um projeto coletivo.

Palavras-chave: convivência, comunidade, liderança e solidariedade, significados. 


\section{Introducción}

En la actualidad, resulta importante reconocer cómo se construyen, organizan y desarrollan procesos de convivencia en las comunidades, pues esto da cuenta de la forma como las personas se vinculan entre sí, con sus entornos y con una realidad social que toma vida precisamente a partir de estas relaciones. El estudio de la convivencia empezó a popularizarse con la publicación de los trabajos de Illich (2003), que planteó el término de "la convivialité", el cual hace referencia a una sociedad que posibilita a las personas ejercer acciones autónomas y creativas, es decir, posibilidades de ser. En España y en América Latina, el tema de la convivencia se ha abordado desde, al menos, dos tendencias. En la primera, la convivencia ha sido asumida como elemento esencial para la construcción de un escenario de paz, en oposición a la violencia, la marginalización y la exclusión generalizada que se vive en muchos de estos territorios (Arango, 2006; Maturana, 1997). En la segunda tendencia, se ha trabajado a partir de los conceptos de interculturalidad, participación ciudadana e inclusión social, derivados de los fenómenos de la migración urbana (Giménez, 2005; Sánchez-Antelo et al., 2008).

La convivencia puede ser entendida como "una relación armoniosa entre gentes que viven juntas; es vivir en buena armonía con los demás. Convivencia es vivir en compañía. Convivencia es tanto la acción de convivir como la relación entre quienes conviven" (Giménez, 2005, p. 8). En este sentido, la solidaridad ya no es solo un dato que describe formas de interacción, sino que es una forma de contrato natural orientado a establecer un nuevo orden social (Serres, 1991). La convivencia promueve un ejercicio participativo que facilita la interacción social, el desarrollo de vínculos, el fomento de la sociabilidad y la colaboración, aspectos que enriquecen la cohesión social y el desarrollo de redes de apoyo (Sánchez y González, 2006).

Por su parte, para Arango (2006), la convivencia es "convivir, es vivir con otros. La convivencia consiste en construir una forma de vivir de manera compartida con otras personas. Y esta forma de vivir se constituye en nuestro mundo personal y nuestra realidad social" (p. 411). Desde esta perspectiva, la convivencia implica entonces una interacción entre sujetos y realidades, traspasada por el establecimiento de vínculos que tienen como fin generar compromisos y acciones orientadas a transformar situaciones compartidas por toda la comunidad. En tal sentido, el ejercicio de la convivencia implica el vivir en relación con un otro, una relación de interdependencia basada en el reconocimiento de derechos y diferencias, la expresión de afecto y empatía, el fortalecimiento de vínculos y el desarrollo de un sentido de reciprocidad que genera patrones de acción compartidos (Maturana, 1997).

No obstante, la convivencia como proceso social ha sido afectada por el creciente individualismo, la politización de las relaciones comunitarias, la visión asistencialista que se propone desde los programas estatales y la falta de cohesión social. En este sentido, el no establecimiento de relaciones de convivencia altera el orden social, al no promover vínculos que permitan a las comunidades crear redes orientadas a satisfacer necesidades comunes y a propiciar condiciones de bienestar psicosocial.

De igual forma, cuando no prevalece la convivencia surge la falta de solidaridad, el egoísmo y la imposibilidad de desplegar una conducta de empatía como factores que facilitan la aparición de la violencia y la indiferencia social. Por ejemplo, los índices de violencia en Cali muestran que la tasa de homicidios se incrementa cada año, pasando de 333 casos en el 2008 a un total de 1939 en el 2013 (cifra suministrada por el Observatorio Social de la Alcaldía de Cali). Estas cifras dan cuenta de una fractura en el orden social, una desvalorización de la vida y del otro, y por lo tanto, en algunos casos, ausencia de convivencia. Esta situación ha llevado a que las instituciones desarrollen programas para la promoción de estilos pacíficos de convivencia y la priorización de valores tales como el respeto, la paz y la tolerancia. Es necesario reinventar una manera distinta de habitar el mundo, de ver las relaciones y de propiciar vínculos traspasados por la asertividad y el respeto (Castells, 1997).

A la vez, es importante - más allá de las regulaciones jurídicas y políticas - dar voz a las comunidades con el fin de que se pronuncien en relación con los significados y las experiencias que viven alrededor de la convivencia, y se reconozcan así como actores sociales y agentes de cambio.

Identificar qué vulnera la convivencia o, por el contrario, cómo se favorece y cómo las personas la conciben, permitirá tener mayor información para fortalecer nuevos recursos de solidaridad que reconozcan la necesidad de la semejanza y la diferencia como componentes insuperables de una vida en común (Melucci, 2001). Tales componentes deben ser mantenidos en un equilibrio aceptable con 
el propósito de posibilitar la supervivencia y la participación ciudadana. En resumen, trabajar sobre la convivencia implica la construcción de una sociedad traspasada por un sentido de justicia social e inclusión, sustentada en la resolución pacífica de conflictos, en la cual las personas se sientan partícipes de los procesos de cambio dentro de sus comunidades, y emerjan vínculos sociales que toman vida a partir de la solidaridad, la empatía y el respeto por la convivencia, sin significar que no exista el conflicto.

\section{Entrando al problema}

Ahora bien, "darle voz" a las comunidades implica adentrarse en la forma como estas construyen significados socialmente compartidos (Hammersley y Atkinson, 1994). El problema del significado se instaura en la psicología actual desde la forma como los sujetos generan conocimientos que les son propios, a través de la interpretación y sentido que dan a sus experiencias. Dichas experiencias son solo posibles en las relaciones diarias con los otros, en las actividades propias de la cotidianidad, enmarcadas en relaciones barriales o comunitarias.

Bruner (1986) propone que la construcción de significados se constituye en un referente fundamental para entender cómo los sujetos construyen su identidad y dan sentido a su experiencia. Para este autor, la construcción de significados está íntimamente ligada con las transacciones - negociaciones-que ejecutan las personas en un espacio cultural determinado. En tal sentido, los significados están situados histórica y contextualmente en cada comunidad, grupo y cultura.

La cultura es como un foro, un espacio de reflexión y discusión en el que las personas tratan de llegar a consensos o bases de acuerdo sobre determinado tema o fenómeno (Bruner, 1998). Lograr el consenso implica un proceso de negociación que toma vida en las relaciones intersubjetivas que desarrollan los sujetos (Bruner, 1986). Desde esta perspectiva, el sujeto, al participar en las interacciones con otro, negocia significados que van a permitirle crear un desequilibrio en sus propias estructuras cognitivas, lo que a su vez genera nuevas formas de asumir una realidad. Es así como para Bruner (2003) solamente, "a través de nuestras propias narraciones es como principalmente construimos una visión de nosotros mismos en el mundo, y es a través de sus narraciones como una cultura ofrece modelos de identidad y acción a sus miembros" (p. 15).
Las narraciones implican al menos un acercamiento al otro, pensado como persona o fenómeno social, un proceso que en palabras de Vásquez (2001), irá construyendo una memoria social, es decir, un proceso de construcción y reconstrucción intersubjetiva, a partir de encuentros discursivos y conversacionales que transcurren en la vida cotidiana. Todas estas relaciones se dan en un espacio comunitario caracterizado por su polisemia y por un orden social que en algunos momentos sugiere su fraccionamiento debido a una sociedad cada vez más individualista.

En opinión de Duque (2010), las comunidades son un espacio vinculante de interrelaciones intencionadas que propenden hacia la satisfacción de necesidades y dan un sentido de inclusión. La comunidad es "un grupo social dinámico, histórico y culturalmente construido y desarrollado, preexistente a la presencia de los investigadores o de los interventores sociales" (Montero, 2004, p. 286). De esa manera, la comunidad emerge como unidad de análisis y de reconocimiento individual y social que permite a los sujetos crear un sentido de vida. Es por ello que la interpretación que hace una comunidad de un suceso puede variar sustancialmente, encontrándose diversos discursos y narraciones según los actores sociales implicados.

Vinculado con lo comunitario, el barrio aparece como un escenario de producción y sentido, al igual que de construcción de identidades, por tratarse de un "espacio simbólico-ideológico y referente de identidades sociales urbanas" (Gravano, 2003, p. 12). El barrio no solo es un espacio geográfico y político administrativo; se trata de un espacio en el que se escenifica la vida cotidiana de las comunidades, y se crean y recrean discursos de sentido, a partir de una serie de prácticas que constituyen identidades colectivas (De Certeau, 2006).

En consecuencia, las dinámicas comunitarias articulan las diferentes experiencias de sus miembros en un entramado de relaciones sociales que modelan sus acciones, representaciones, imaginarios y proyectos de vida. Siguiendo a Torres-Carrillo (2009), los vínculos de vecindad van fortaleciendo interacciones de intercambio y reciprocidad, desde la constitución de lazos de pertenencia, experiencias compartidas, apropiación del territorio y relaciones de solidaridad.

\section{Método}

Para abordar la pregunta de investigación, se acudió a un enfoque cualitativo con diseño etnográfico, 
orientado a establecer desde la cotidianidad de los líderes y su interacción con la comunidad los significados que construyen en torno a la convivencia. El alcance del estudio fue descriptivo, apuntando a caracterizar desde la visión de los líderes, sus narrativas e imaginarios compartidos (Hammersley y Atkinson, 1994; Sandoval, 1997).

\section{Participantes}

En la investigación participaron un total de cinco (5) líderes comunitarios de la comuna 18 de Santiago de Cali, Colombia, asentamiento popular ubicado al sur de esta ciudad. Los líderes activos estaban vinculados a la junta de acción comunal y llevaban a cabo proyectos de desarrollo para su comunidad. Con respecto a algunas características sociodemográficas de los participantes, las edades comprendían entre los 35 y los 57 años, todos habían vivido en el barrio entre 7 y 17 años, y ocupaban cargos en la junta de acción comunal. La población inicial contemplaba un número mayor de sujetos, pero se llegó a un punto de saturación en el cual no se encontraron datos adicionales para el desarrollo del contenido de las categorías propuestas, y tampoco se lograron nuevos hallazgos (Denzin, 1978).

\section{Instrumentos}

Los instrumentos utilizados para la construcción de los datos fueron la entrevista semiestructurada, el grupo de discusión y el ejercicio de foto-voz. La entrevista semiestructurada se elaboró a partir de una serie de preguntas orientadoras, previamente estructuradas y diseñadas (Mayan, 2001). Para su desarrollo, se establecieron ocho (8) categorías analíticas fundamentadas en las ocho (8) dimensiones de análisis propuestas por Giménez (2005), presentadas en la tabla 1.

La técnica foto-voz es una herramienta de la investigación que utiliza la toma de fotografías como recurso para identificar y representar diversos aspectos de la realidad de una comunidad (Wang, 1999). La implementación de este tipo de instrumentos promueve la reflexión sobre los saberes construidos colectivamente y un diálogo crítico sobre la convivencia, como se propone en este caso.

Por su parte, el grupo focal permitió obtener información específica y colectiva sobre los significados relacionados con la convivencia. Esta metodología hizo posible conocer aspectos de la vida de
Tabla 1

Definición de dimensiones

\begin{tabular}{|c|c|c|}
\hline $\mathrm{N}^{\mathrm{o}}$ & Dimensiones & Aspectos centrales \\
\hline 1 & Relacional & $\begin{array}{l}\text { La existencia o no de la interacción } \\
\text { entre los sujetos, y la naturaleza de esa } \\
\text { interacción. }\end{array}$ \\
\hline 2 & Normativa & $\begin{array}{l}\text { Normas compartidas; conocimiento y } \\
\text { aceptación de las normas; adecuación } \\
\text { normativa. }\end{array}$ \\
\hline 3 & Axiológica & $\begin{array}{l}\text { Valores y finalidades compartidas; } \\
\text { reconocimiento y respeto de lo no } \\
\text { compartido. }\end{array}$ \\
\hline 4 & Participativa & $\begin{array}{l}\text { Presencia en los ámbitos decisorios e } \\
\text { implicación de todos en la vida social } \\
\text { conjunta. }\end{array}$ \\
\hline 5 & Comunicacional & $\begin{array}{l}\text { Comunicación existente; pautas y } \\
\text { espacios de comunicación y eficacia. }\end{array}$ \\
\hline 6 & Conflictual & $\begin{array}{l}\text { El tratamiento de la conflictividad } \\
\text { latente y manifiesta; comportamientos } \\
\text { pacíficos y violentos, y negociación } \\
\text { versus intervención de terceros. }\end{array}$ \\
\hline 7 & Actitudinal & $\begin{array}{l}\text { Respeto hacia el otro y naturaleza de } \\
\text { la tolerancia; voluntad de inclusión o } \\
\text { exclusión. }\end{array}$ \\
\hline 8 & Identitaria & $\begin{array}{l}\text { Identidades compartidas y no com- } \\
\text { partidas; sentido de pertenencia. }\end{array}$ \\
\hline
\end{tabular}

Nota. Tomado de "Convivencia: conceptualización y sugerencias para la praxis”, por C. Giménez, 2005, en Puntos de Vista: Cuadernos del Observatorio de las Migraciones y la Convivencia Intercultural de la Ciudad de Madrid, 1, p. 14.

las personas, y su contexto cultural más inmediato (Sandoval, 1997).

\section{Procedimiento}

La información recaudada se sometió a un proceso de triangulación de datos al poner en acción diferentes instrumentos (Denzin, 1978). La codificación se realizó a través del software de análisis cualitativo asistido por computadora (QDA) ATLAs.ti. Este programa de análisis posibilitó la organización conceptual de los datos obtenidos y la creación de relaciones de diferentes tipos entre los códigos, a partir de representaciones gráficas que permiten la conceptualización del trabajo.

\section{Resultados}

Gracias a la codificación de los datos en el software ATLAs.ti fue posible establecer el número de citas que 
corresponden a cada una de las categorías abordadas (véase figura 1). La categoría de Participación tuvo el mayor número de citas con un total de 135; mientras que la categoría Relacional tuvo 50 citas. Esto da cuenta de cómo para los líderes de la junta de acción comunal la convivencia es posible en la medida en que la comunidad y ellos mismos participen en los proyectos que se realizan en el barrio, constituyéndose la participación en un tema central.

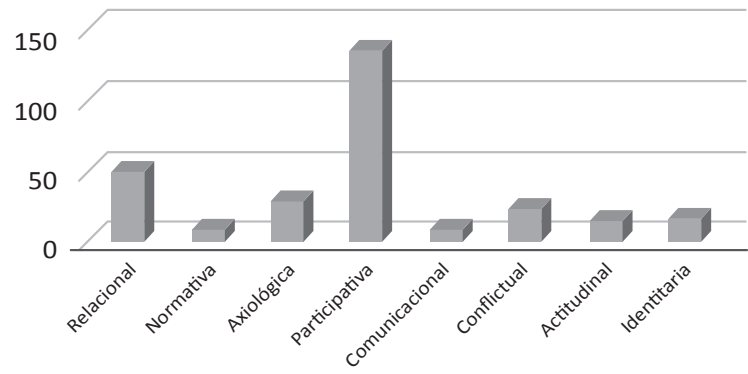

Figura 1. Número de citas por categorías analíticas. Elaboración propia.

A continuación se presenta la red de relaciones asociadas a cada una de las categorías de la investigación.

En la categoría Relacional se establecieron tres elementos fundamentales para el análisis de los datos: la interacción, la empatía y la comunicación. La interacción entre los líderes y la comunicación se ven fragmentadas por el individualismo y el egoísmo, las cuales se orientan a la satisfacción de necesidades propias, sin tener en cuenta las de los demás. En las relaciones construidas, prevalece el individualismo; la comunidad suele unirse solo ante eventos extremos como la muerte de una persona o un desastre natural, pero la relación finaliza con la prestación de una ayuda que pareciera reproducir la visión asistencialista a la que se han visto abocados, y también refleja un proceso identitario, en el cual lo que sucede no me gustaría padecerlo. Esto se relaciona directamente con la imposibilidad de generar un proceso empático y de situarse en el lugar de otro.

El mismo desinterés de la gente ha hecho que el barrio no avance, la gente solo le interesa invertir en su vivienda y unas cuadras se han unido y han pavimentado sus cuadras, pero otras no. Entonces eso es lo que limita el desarrollo como barrio pero también como persona. Porque si cada uno no pone un poquito para mejorar así no tengamos el apoyo del municipio con todas esas necesidades que tenemos de predios, de servicios, entonces ahí estamos reflejando que no nos gusta el orden y mejorar sino que, pues si se desbarata, que se desbarate (Comunicación personal, 5 de abril del 2014).

Las dificultades relacionales parecen verse reflejadas en la categoría Normatividad que se presenta en la figura 2, en la cual se evidencia que dentro de la comunidad no se construyen normas explícitas; por el contrario, prima una serie de normas implícitas compartidas social y culturalmente, preexistentes y matizadas por las experiencias de cada sujeto.

En la categoría Axiológica (véase figura 3), se identificaron aquellos valores que guían y orientan a los líderes y a la comunidad. La solidaridad aparece como un valor fundamental para pensar la convivencia, pero ausente la mayoría de las veces en las relaciones cotidianas de las personas. Los líderes consideran que la solidaridad puede posibilitar que una comunidad busque el bien común y se mantenga unida; sin embargo, al analizar la solidaridad desde el barrio aparece una serie de antivalores tales como la apatía, el egoísmo y el individualismo. Llama la atención que en lo expuesto por los líderes pareciera no haber una aceptación por las diferencias y poca tolerancia ante las opiniones de los demás. Esto sin duda influye en la participación que las personas puedan tener en la comunidad.

En la categoría Participación comunitaria (véase figura 4), se evidenció constantemente la idea de que la participación es la estrategia primordial para ayudar a mejorar las condiciones de vida de la comunidad, con acciones que promuevan el desarrollo del sector. De igual forma, surge en lo narrado por los líderes la relación comunidad-Estado como una categoría emergente. Relación de tensión y conflicto, la cual refleja la ruptura entre ambas por el incumplimiento de promesas, pero a la vez se reconoce importante por el deseo de obtener beneficios que se pueden lograr mediante la participación electoral y desde un marco jurídico-político.

En general, la participación es poco frecuente por parte de la comunidad; los líderes solo ejecutan acciones específicas que obedecen a demandas cotidianas y que no se articulan necesariamente a proyectos macro. La participación se ve limitada por el individualismo, la apatía y la desconfianza de la comunidad hacia la gestión de la junta debido a la politización de las relaciones. La participación se da en función de la satisfacción de necesidades básicas y de 


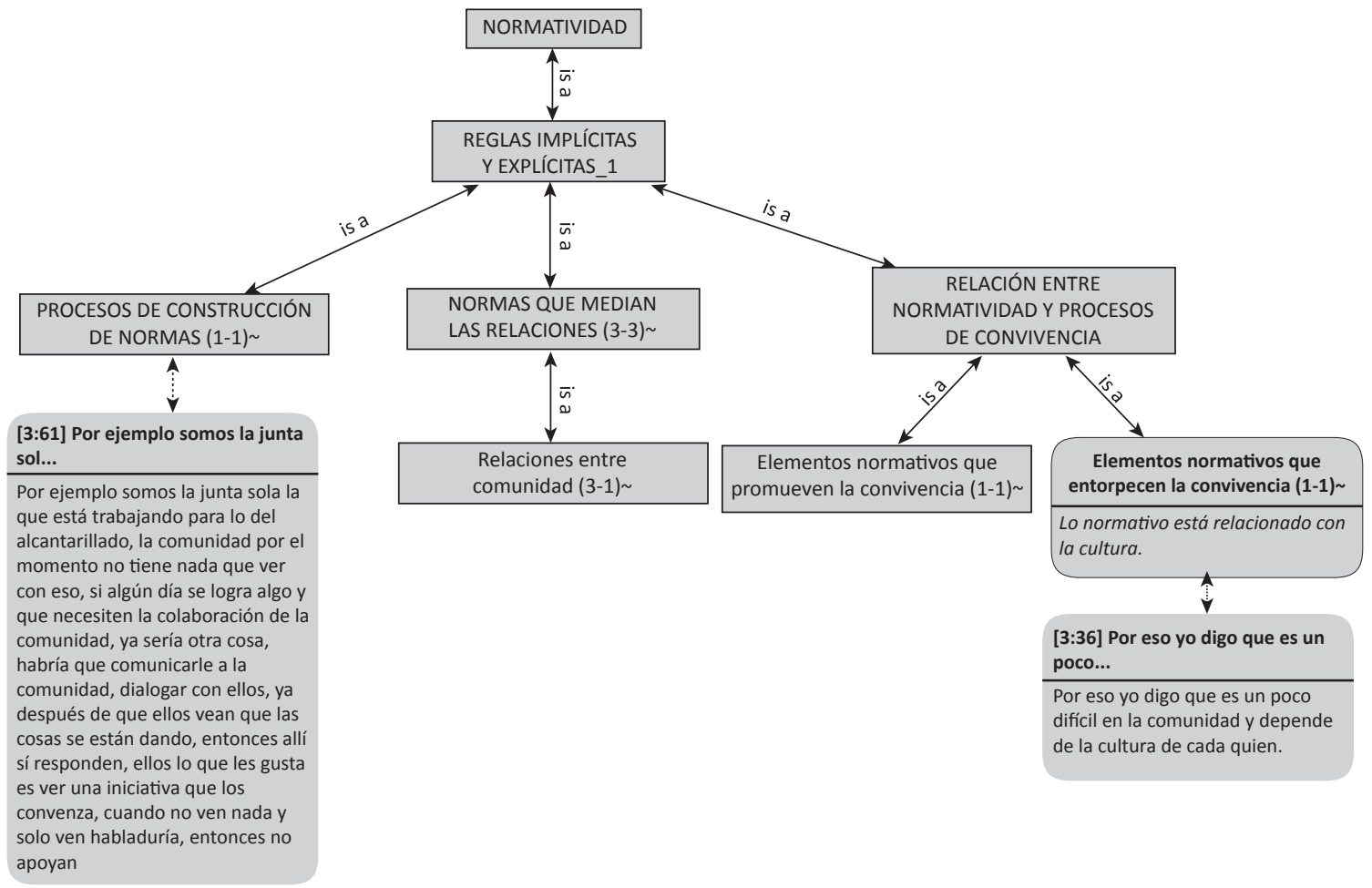

Figura 2. Representación de la red de la categoría Normatividad. Elaboración propia.

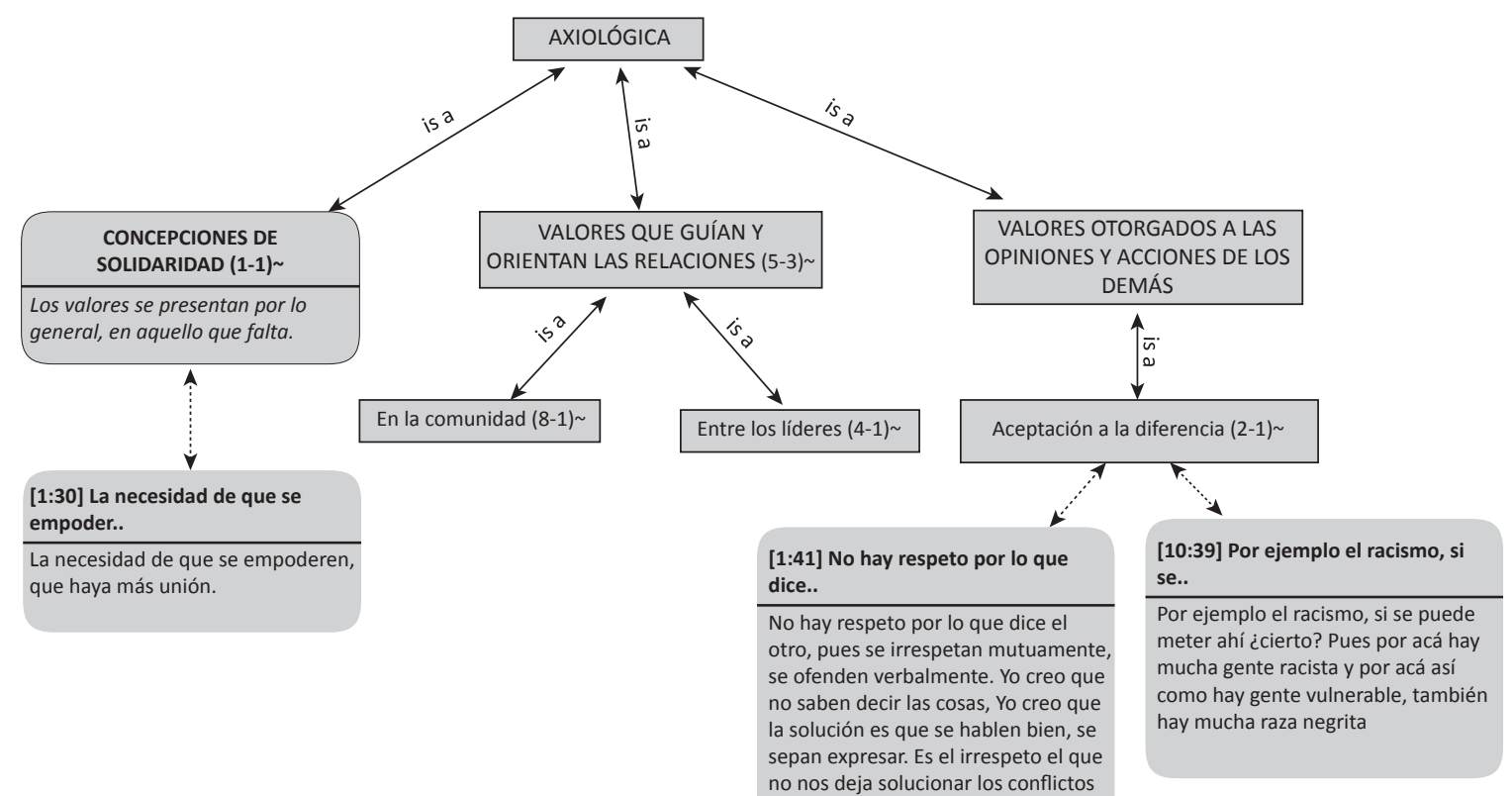

Figura 3. Representación de la red de la categoría Axiológica. Elaboración propia. 


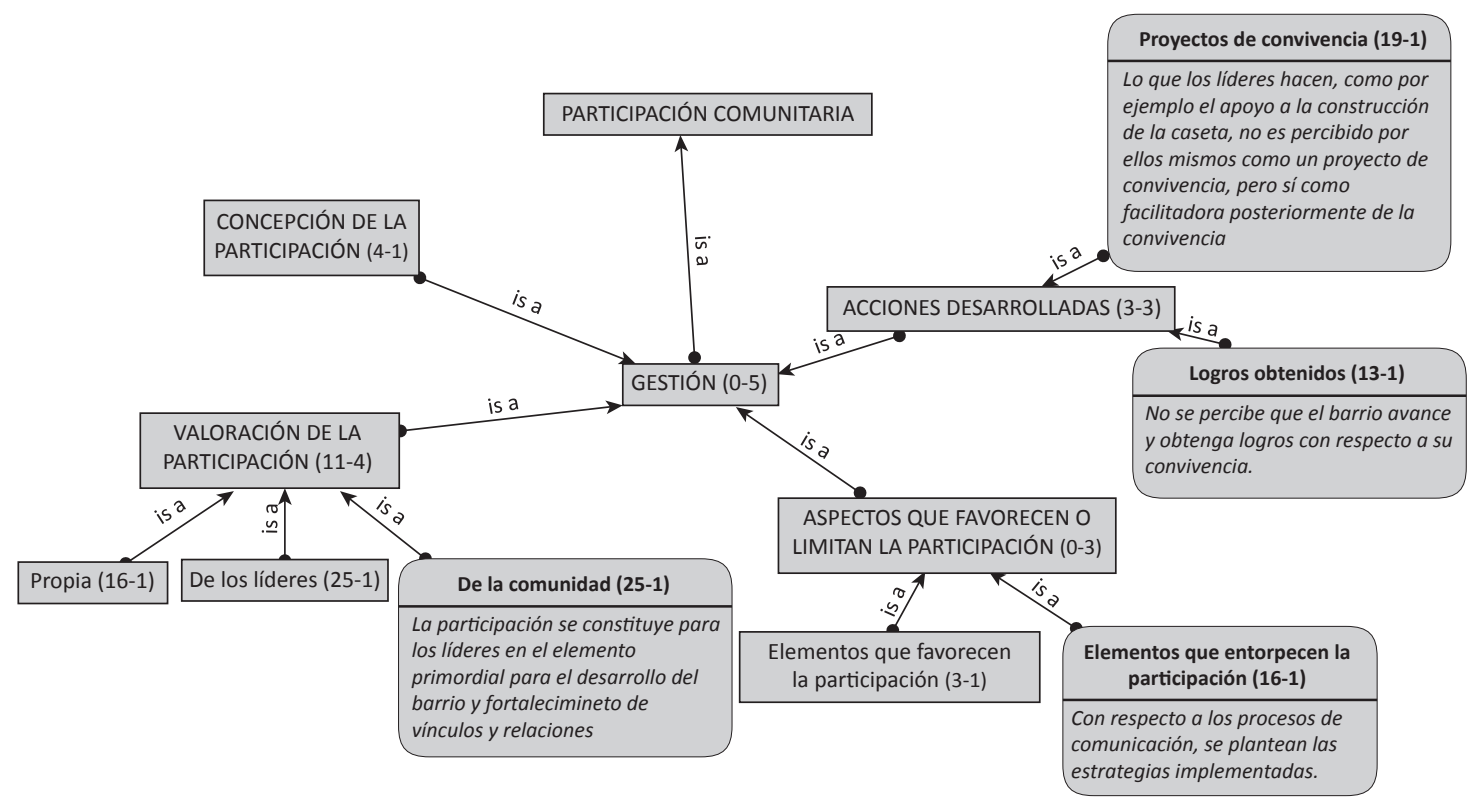

Figura 4. Representación de la red de la categoría Participación comunitaria. Elaboración propia.

infraestructura física, y la obtención de ganancias que se legitiman desde una visión asistencialista que promueve el recibir y obstaculiza la autogestión. En este sentido, uno de los lideres manifestó: "La gente no quiere participar, no quiere involucrarse en nada que se propone. Nadie es capaz de hacer algo, solo les preocupa lo que ellos necesitan" (Comunicación personal, 10 de abril del 2014).

En relación con la resolución de conflictos - categoría conflictual-, se exploró sobre las situaciones que pueden ser negociadas en la comunidad y cómo son resueltos los conflictos. En segunda instancia, se revisaron las estrategias que han desarrollado los líderes para la toma de decisiones. A este respecto, no hay una evidencia amplia de una negociación de conflictos que parta de una propuesta colectiva; en tal sentido el Estado y sus instituciones emergen como entes reguladores exclusivos. En la mayoría de los casos, se recurre a los jueces de paz o a procesos de conciliación ante instituciones gubernamentales. De igual forma, muchos temas propuestos en las reuniones de junta terminan en discusiones que no llegan a acuerdos explícitos.

En la categoría actitudinal, se concibe por parte de los líderes fundamentalmente el respeto por el otro y la posibilidad de favorecer procesos inclusivos. No obstante, prevalece la coexistencia que se orienta en algunos casos a la agresión enmascarada vista por el no reconocimiento del otro, la falta de comunicación, la interacción y la empatía. Y en la categoría identitaria, el rol que ejercen como líderes genera una noción de identidad ligada a un antes y a un después de cumplir con sus funciones dentro de la junta. El ser líderes les ha permitido el desarrollo de habilidades personales y sociales, además de crear contactos con otras personas, pero ha obstaculizado el desarrollo de tareas domésticas que pueden afectar en algún momento sus relaciones familiares.

Asimismo, desde la codificación del software ATLAS.ti se estableció la co-ocurrencia entre cada una de las categorías, es decir, las codificaciones que describían dos categorías al mismo tiempo. Para este ejercicio, se revisaron cinco de las categorías abordadas, pues entre ellas se presentó mayor número de codificaciones entrecruzadas.

En la tabla 2, se puede observar, por ejemplo, cómo las categorías Axiológica-Valores y Participación se cruzan con la categoría Relacional con una frecuencia de nueve y diez casos, respectivamente, pues para los líderes la percepción que la comunidad tiene de ellos es fundamental en muchas ocasiones para gestionar o no proyectos en el barrio. A este respecto, los participantes manifestaron que la comunidad no confía en su gestión, y en muchas ocasiones solo participa cuando cree que pueden obtenerse beneficios concretos. Para los líderes, la comunidad incrementa sus conductas de participación de acuerdo con la consecución de estímulos materiales, consolidándose y manteniéndose así una relación de dependencia o una visión asistencialista; de allí que la 
Tabla 2

Relación de categorías (co-ocurrencia)

\begin{tabular}{cccccc}
\hline Categorías & Axiológica & Normatividad & Participación comunitaria & Conflictual & Actitudinal \\
\hline Relacional & 9 & 0 & 10 & 4 & 3 \\
Normatividad & 1 & & 2 & 1 & 0 \\
Conflictual & 1 & 1 & 1 & & 0 \\
Actitudinal & 4 & 0 & 6 & 0 & \\
Identitaria & 0 & 0 & 3 & 0 & 0 \\
\hline
\end{tabular}

Nota. Elaboración propia.

convivencia no emerja como un proceso natural en las relaciones que se entablan.

\section{Discusión de resultados}

Las concepciones de los líderes sobre la convivencia giraron principalmente en torno a: 1) la solidaridad; 2) la evitación de conflictos; 3) la expresión de afecto; 4) la construcción de acuerdos; 5) el respeto por el otro y 6) la colaboración.

La convivencia fue definida por los líderes como aquellos procesos que hacen posible la construcción y el establecimiento de acuerdos que garantizan la consolidación de buenas relaciones en la comunidad y evitar los conflictos. Esta concepción solo puede operar en la medida en que la solidaridad sea el valor que guíe y oriente las relaciones, y que la participación comunitaria sea efectiva. Al respecto, los líderes plantearon: "Tener buenas relaciones con los vecinos, que lleguemos a acuerdos en muchas cosas, para evitar conflictos, ponerse de acuerdo en todo lo que se vaya a hacer en el sector y hacerlo bien" (Comunicación personal, 12 de diciembre del 2013).

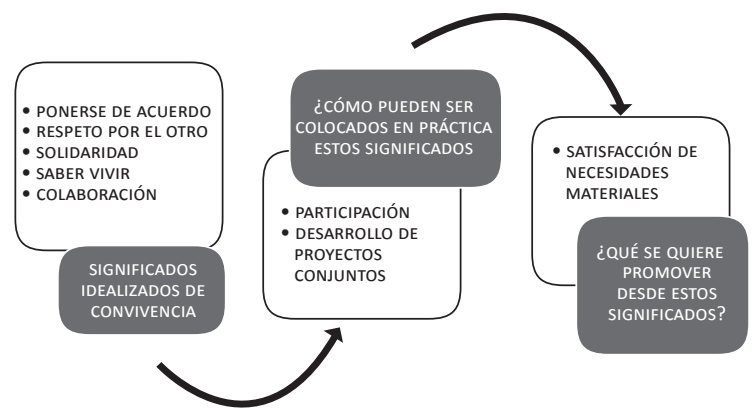

Figura 5. Representación de los significados de convivencia. Elaboración propia.
Según los líderes, aquellos mecanismos y estrategias que pueden posibilitar su aplicación y puesta en marcha en la comunidad son posibles desde el fortalecimiento de la participación y la posibilidad del desarrollo y construcción de proyectos conjuntos. Estas acciones de convivencia y solidaridad están centradas exclusivamente en la satisfacción de necesidades materiales, tales como la construcción de muros de contención, canaletas para el paso de aguas residuales y la atención de algunas familias en casos concretos de calamidad doméstica.

$\mathrm{Al}$ respecto, otro líder apunta:

Por ejemplo si un vecino requiere o está en una situación difícil de salud con un hijo, entonces uno le puede orientar, entonces uno le dice: puede ser el vecino de otra cuadra pero se acercan a uno, se dan cuenta que uno hace cosas. Entonces uno les puede orientar, entonces yo creo que eso es ser solidario. Entonces también si tiene una situación económica se puede ser solidario desde las cosas que se tengan (Grupo focal, 27 de noviembre del 2013).

Además, un elemento que hizo parte de la definición de la convivencia fue que en algunos casos la existencia o construcción de un vínculo afectivo se constituía en una condición necesaria para su desarrollo y mantenimiento, principalmente con los familiares y vecinos cercanos. Acerca de ese tema, uno de los líderes expresó:

Porque yo veo un vecino mal y yo veo cómo le puedo colaborar no en plata porque en plata no (sonríe). Por ejemplo, necesita la colaboración de un trabajo para ver si le ayudan un préstamo, pues se hace la forma de conseguírselo. No pues, para apoyar de todas las maneras asistiendo cuando lo invitan a uno a las reuniones que haiga [sic] y en fin, es la única forma que 
yo veo que podemos apoyar (Comunicación personal, 10 de abril del 2014).

La conducta de solidaridad termina en el acto mismo de ayuda, y no se extiende a otras experiencias comunitarias que promuevan la cohesión social. Por tanto, si la solidaridad no está presente efectivamente en una comunidad, en un sentido real de vínculo social se pueden ver fracturados todos aquellos procesos que posibiliten relaciones de convivencia.

La solidaridad como valor clave en las relaciones barriales se concibe como algo muy preciado, pues desde esta es posible la construcción de vínculos fuertes. Podría pensarse entonces en la solidaridad como un recurso no convencional que posibilita el desarrollo social. A este respecto, Max-Neef, Elizalde y Hopenhayn (1986) plantean que "los recursos no convencionales potencian un desarrollo que va más allá de la noción convencional de acumulación (aun cuando la incluye), ya que se funda, además, en el acervo del saber práctico generado por la propia comunidad" (p. 55).

Sin embargo, esta concepción de solidaridad dista mucho de las relaciones y valores que cotidianamente definen y consolidan estos procesos, pues para los lideres el individualismo, la apatía, el egoísmo y las rivalidades imperantes en el barrio limitan la participación y, por consiguiente, el establecimiento de relaciones de solidaridad, la negociación de conflictos y la toma de decisiones. El individualismo imperante y la desconfianza no permiten la inclusión ni el desarrollo de tendencias dirigidas al bien de toda la comunidad. Uno de los participantes afirmó:

La convivencia muy regular. No sé porque se está dando así, es muy regular, parece egoísmo. Alguien quiere hacer algo en el barrio y los demás dicen que ya la gente se va a hacer rico en el barrio, es como egoísmo envidia (Comunicación personal, 3 de diciembre del 2014).

\section{Otro de los participantes estableció:}

Eso individualista nos ha afectado bastante porque aunque nos conocemos, no somos unidos, para decir vamos a mejorar aquello, lo otro no, eso hace que si alguno puso un empeño más, y otro vecino no hace, entonces dice, no pues como ayuda, no le vamos a hacer nada, entonces se van, no hago porque el vecino no apoya (Comunicación personal, 5 de abril del 2014).
Con base en los anteriores y en otros testimonios, los significados de la convivencia se construyen desde dos dimensiones. La primera, relacionada con aquello que no es convivencia, que no la facilita, que la fractura - como el individualismo, la apatía y la poca participación-; tal vez por ello surgen visiones idealizadas de la convivencia que se convierten, más que en una realidad, en un objetivo por alcanzar.

En una segunda dimensión, y a falta de una significación de convivencia construida desde la proactividad de las acciones, emerge el espacio familiar como lugar idóneo para pensar y sustentar la interpretación de la convivencia, a partir de experiencias familiares interiorizadas en las primeras etapas de la vida. En tal sentido, Arango (2006) plantea que la convivencia da cuenta de la relación que los sujetos generan con dimensiones más amplias en las cuales están inscritos, tales como la cuadra, el barrio, la comuna, la ciudad, el país y la normatividad.

Yo no soy muy unido con la junta, pero con mi familia sí; todos los días todos hacen las cosas unidos. Yo les digo eso se hace en casa y cuando las cosas se hacen en casa entonces se refleja afuera, entonces eso le digo yo a la gente, que hay que aprender eso (Comunicación personal, 10 de abril del 2014).

Las dificultades experimentadas por la comunidad en torno a la convivencia se deben a varios factores, entre los que se encuentran principalmente los relacionados con los procesos comunicativos. En este caso, la comunicación se valora de forma negativa, aspecto que afecta la interacción de las personas, pues al romperse la comunicación se ve resquebrajada la interacción. La comunicación solo es fluida cuando se dan refuerzos inmediatos ante situaciones de desventaja social que necesitan ser satisfechas.

De igual forma, el individualismo imposibilita a las personas generar comportamientos empáticos que los posicione de tal forma que puedan comprender el actuar del otro, aspecto básico de la convivencia y la comunicación. En la denominada "crisis de la modernidad", el orden social se ha visto afectado ante la ruptura de los lazos sociales y el incremento de las fisuras en el tejido social (Carballeda, 2002). Es así como en algunos contextos, los sujetos adquieren una visión más individualizada de los procesos, primando lo "mío", y lo "tuyo" es casi inexistente. Esta visión resulta preocupante para una psicología comunitaria, y pone en tela de juicio nociones comunes que manifiestan que es precisamente en estos barrios 
donde se consolidan mayores lazos de unión y convivencia con el fin de responder a problemas puntuales de la comunidad.

Si la interacción no se da, se empiezan a tener problemas en la cohesión social y en la afiliación de las personas al grupo, pues el sujeto participará de él en la medida en que se sienta identificado con sus acciones y prácticas, y pueda discernir entre quién es él y su posición frente al grupo (Moreland, 1987). Y aunque la cohesión social no garantiza por si sola el desarrollo de procesos de convivencia (Palou, Rodríguez y Vilá, 2013), sí se constituye en factor que la puede facilitar, ya que la convivencia no se puede construir desde el individualismo; por el contrario, el hombre necesita la presencia de otros para existir (Arango, 2006).

La cohesión social depende de elementos como la condición socioeconómica, pero sobre todo de las trayectorias y experiencias compartidas de los grupos (Garavito, 2009). Esto es particular en el sector en el que se realizó la investigación, pues si bien hay una historia de lucha y de afianzamiento de un territorio - pese a la amenaza inicial del Estado por desalojarlos de los predios-, cada vez hay más división $\mathrm{y}$ fragmentación social.

La falta de cohesión social y el individualismo demuestran que en el barrio existen más procesos de coexistencia que de convivencia, en la medida que en la coexistencia (Giménez, 2005) no hay una implicación verdadera con el otro, ni un sentido de reciprocidad y responsabilidad compartidas; más que una verdadera interacción, prima el contacto fugaz, sustentado en un interés pragmático.

Giménez (2005) considera que la coexistencia es un proceso intermedio entre la convivencia y la hostilidad:

Así, una situación de coexistencia pacífica, sólida, trabajada y de larga duración entre dos grupos étnicos deberá tener rasgos claros de convivencia. En el otro lado del continuum una coexistencia precaria, de baja calidad, con contactos de agresividad, está ya en las puertas de la hostilidad (p. 82).

Otros factores que parecen influir para el no desarrollo de la convivencia son la politización de las relaciones, y la visión asistencialista:

Porque uno aquí se desobliga, fuera que le fuera a pagar a la gente entonces sí. La gente desobliga, gente hay muchísima y como le digo cuando es para repar- tir algo entonces la gente cae, pero [si] es para colaboración son pocos (Comunicación personal, 27 de noviembre del 2013).

Se destaca en los resultados de la investigación la transformación que sufren las mujeres líderes a nivel personal. Pese a que casi todas han tenido dificultades familiares por el manejo del tiempo dentro de sus hogares, se han enriquecido en el desarrollo de relaciones sociales, la visión que tienen de sí mismas y la valía personal. Al respecto, Morote (2011) plantea que precisamente esta división de roles de las mujeres líderes sociales devela cómo a pesar de las condiciones de pobreza en algunos territorios, en las comunidades, y especialmente en las mujeres, estas logran traspasar los límites impuestos por los contextos.

Ser líder me ha cambiado la vida, porque ya me he hecho una persona diferente. Yo siempre soy que primero tú y después yo. Antes era así, ahora aprendí que para estar bien, primero tengo que estar bien conmigo misma y le brindo eso a mi comunidad. De acuerdo como yo me sienta, así mismo yo me comporto con la comunidad. En el trabajo comunitario he ganado, conocer a mucha gente, relacionarme más, estar más despierta, mejorar mi comportamiento (Comunicación personal, 9 de abril del 2014).

\section{Conclusiones}

Los significados de convivencia obedecen a una perspectiva idealizada de los líderes que no corresponde a la realidad imperante en la comunidad. La solidaridad como valor esencial para la convivencia fue definida como aquello que posibilita que una comunidad busque el bien común y que esté unida. Sin embargo, cuando se pregunta sobre cómo esta definición opera en el barrio, los líderes plantearon que no es una constante en las relaciones, pues más que la solidaridad priman valores tales como el egoísmo, el individualismo, la desunión y la apatía, tanto en la comunidad como dentro de la junta de acción comunal. Al respecto, Elías (1990) se pregunta cómo es posible un ordenamiento de la convivencia que permita un mejor equilibrio entre las necesidades personales y el funcionamiento social. El mismo autor responde que dicho equilibrio puede sustentarse cuando los sujetos estén satisfechos y la sociedad libre de trastornos, tensiones y luchas. Sin embargo, y en sus palabras, "el abismo entre individuo y sociedad que se abre una y otra vez ante nuestro pensamiento guarda 
una estrecha relación con las contradicciones entre requerimientos sociales y necesidades particulares" (p. 23). Esto no deja de ser paradójico si se tiene en cuenta que, "los seres humanos caminan por calles aparentemente ajenas e independientes de los demás, ligados a otras personas por un cúmulo de cadenas invisibles, ya sean estas cadenas impuestas por el trabajo, o por propiedades, por instintos o por afecto" (p. 29).

Por su parte, la identificación de "prácticas de convivencia comunitaria" se encuadra ante situaciones de ayuda matizadas por circunstancias que generan altos niveles de emotividad, como, por ejemplo, desastres naturales. Sin embargo, estos encuentros terminan cuando se trata de dar solución a la situación. En general, no se dan procesos participativos sustentados en la formulación de proyectos, debido —según los líderes- a experiencias negativas con las juntas de períodos anteriores, al no establecer resultados concretos e inmediatos. Las relaciones en la junta de acción comunal no distan de las surgidas en la comunidad, pues para los líderes estas se caracterizan igualmente por la escasa participación y las rivalidades existentes que no facilitan la construcción de proyectos comunes. Igualmente, otros factores que promueven la poca participación y la no ejecución de procesos se sustentan en gran medida en una visión asistencialista que ha primado en la comunidad.

Se estableció que las negociaciones de los significados sociales en torno a la convivencia se dan ante experiencias familiares y ante el contacto con realidades sociales en las que priman valores contrarios a la convivencia, como el egoísmo y la apatía; en tal sentido, la negociación de significados se da desde la ausencia y la falta de participación de la comunidad.

Aunque la reivindicación de derechos y la búsqueda de satisfacción de necesidades básicas de las comunidades son aspectos fundamentales para la movilización de un sector o barrio, esto no determina automáticamente el desarrollo de procesos de convivencia. La construcción de dinámicas y situaciones de convivencia no es una cuestión natural; se requiere la formación de los líderes y la comunidad en general en esta temática. Por esta razón, una apuesta clave de transformación social es el desarrollo de programas de formación que posibiliten trabajar sobre los procesos de convivencia y la comprensión de las dinámicas que se dan en el barrio.

De otra parte, los líderes no evidencian en las personas de la comunidad una participación activa, aspecto que puede ser explicado desde el concepto de democracias delegadas, el cual enuncia que "los electores eligen al líder y delegan en él toda la responsabilidad, luego se desentienden casi por completo de la política" (Jiménez-Domínguez, 2008, p. 61). Ante esta perspectiva, no se da una verdadera participación comunitaria; es decir, y en palabras de Montero (2004), no se da una actuación colectiva "en el sentido de aportar (ideas, recursos, materiales) y de comprometerse con el sentido, dirección y finalidad de la acción, a la vez que se obtienen beneficios a partir de los logros colectivos" (p. 141). La escasa participación y los bajos niveles de interacción impiden la negociación de significados y la construcción de acuerdos y consensos. La negociación de significados se da desde la ausencia y la falta de participación de la comunidad, lo que lleva a una idealización de la convivencia, la cual está ausente en las interacciones sociales del barrio.

\section{Referencias}

Arango, C. (2006). Psicología comunitaria de la convivencia. Cali: Programa editorial Universidad del Valle.

Bruner, J. (1986). Realidad mental: mundos posibles. Barcelona: Gedisa.

Bruner, J. (1998). Actos de significado. Más allá de la revolución cognitiva. España: Paidós.

Bruner, J. (2003). La fábrica de historias. Derecho, literatura, vida. Buenos Aires: Fondo de Cultura Económica.

Carballeda, A. (2002). La intervención en lo social. Buenos Aires: Paidós.

Castells, M. (1997). El poder de la identidad. Madrid: Paidós.

De Certeau, M. (2006). La invención de lo cotidiano 2. Habitar, cocinar. México: Universidad Iberoamericana, Instituto Tecnológico y de Estudios Superiores de Occidente.

Denzin, N. (1978). The research act. A theoretical introduction to sociological methods. New York: McGraw-Hill.

Duque, J. (2010). Saberes aplicados, comunidades y acción colectiva. Una introducción al trabajo comunitario. Cali: Programa editorial Universidad del Valle.

Elías, N. (1990). La sociedad de los individuos. Barcelona: Península.

Garavito, J. (2009). Trayectorias de convivencia. Mujeres en un proceso de reconstrucción post-desastre (Tesis de maestría). Universidad del Valle, Cali, Colombia.

Giménez, C. (2005). Convivencia: conceptualización y sugerencias para la praxis. Puntos de Vista: Cuadernos del Observatorio de las Migraciones y la Convivencia Intercultural de la Ciudad de Madrid, 1, 7-31. 
Gravano, A. (2003). Antropología de lo barrial. Estudios sobre producción simbólica de la vida urbana. Buenos Aires: Espacio editorial.

Hammersley, M. y Atkinson, P. (1994). Etnografía: métodos de investigación. Barcelona: Paidós.

Illich, I. (2003). La convivialité. París: Fayard.

Jiménez-Domínguez, B. (2008). Subjetividad, participación e intervención comunitaria. Una visión crítica desde América Latina. Buenos Aires: Paidós.

Maturana, H. (1997). Emociones y lenguaje en educación y política. Santiago de Chile: Ed. Dolmen Ensayo.

Max-Neef, M., Elizalde, A. y Hopenhayn, M. (1986). Desarrollo a escala humana: una opción para el futuro. Santiago, Chile: Cepaur.

Mayan, M. J. (2001). Una introducción a los métodos cualitativos: Módulo de entrenamiento para estudiantes y profesores. Alberta, Canadá: Qual Institute Press.

Melucci, A. (2001). Vivencia y convivencia. Teoría social para una era de la información. Madrid: Trotta.

Montero, M. (2004). Introducción a la psicología social comunitaria. Buenos Aires: Paidós.

Moreland, R. L. (1987). The formation of small groups. En C. Hendrick (ed.), Group processes (pp. 80-110). Newbury Park: Sage.

Morote, R. (2011). Vidas que se hacen historia. Subjetividad y empoderamiento, dos generaciones de mujeres líderes sociales. Perú: Instituto Bartolomé de las Casas, Editor Centro de estudios y publicaciones (CEP).
Observatorio Social de Cali. (2013). Informes estadísticos. Recuperado de: http://www.cali.gov.co/observatorios/ publicaciones.php?id=2538

Palou, B., Rodríguez, M. y Vilá, R. (2013). Convivencia y participación de jóvenes en Sant Boi de Llobregar (Barcelona). Revista de Educación, 361, 1-17. doi: 104438/1988-592X-RE-2011-361-142.

Sánchez, L. y González, J. R. (2006). Estilos de vida y participación comunitaria. La calidad de vida como finalidad. VDP. Venezuela: Soluciones Gráficas.

Sánchez-Antelo, V., Plana, M., Taleb, F., Ferreté, I., Hammich, H., Muñir, M., et al. (2008). Gramáticas de la convivencia, estudio sobre la convivencia en la Salut y Sant Joan de Llefiá, Badalona. Polis, 7(20), 133-150. doi: http:// dx.doi.org/10.4067/S0718-65682008000100008.

Sandoval, C. (1997). Investigación cualitativa. Bogotá: Icfes/ Ascun.

Serres, M. (1991). El contrato natural. Valencia: Pre-Textos.

Torres-Carrillo, A. (2009). Acción colectiva y subjetividad. Un balance desde los estudios sociales. Folios, 30, 5174.

Vásquez, F. (2001). La memoria como acción social. Relaciones, significados e imaginario. Barcelona: Paidós.

Wang, C.C. (1999). Photovoice: a participatory action research strategy applied to women's health. Journal of Women's Health, 8(2), 185-192. doi: 10.1089/jwh.1999.8.185. 